\title{
On the origin of the giant magnetic moment of the Al-Mn quasicrystals
}

\author{
V.S. Kraposhin ${ }^{1}$, D.A. Bazhanov ${ }^{2}$, and P.V. Bocharov ${ }^{1}$ \\ ${ }^{1}$ Bauman Moscow State Technical University, Department of Machinery Technology, Moscow, Russia \\ ${ }^{2}$ Moscow State University, Department of Physics, Moscow, Russia
}

\begin{abstract}
A b$ initio calculations of magnetic moments for icosahedral clusters contained in crystal structures $\mathrm{Al}_{10} \mathrm{Mn}_{3}, \mathrm{Al}_{5} \mathrm{Co}_{2}, \mathrm{Al}_{17} \mathrm{Mn}_{4}\left(\mathrm{Al}_{13} \mathrm{Cr}_{4} \mathrm{Si}_{4}\right.$-type) fulfilled in the framework of Density Functional Theory. The AlMn cluster having the trigonal $D_{3 h}$ symmetry with the triangle of $\mathrm{Mn}$ ions in the interior has the moment being equal to three magnetic moments of a single manganese ion $\left(4.4 \mu_{\mathrm{B}}\right)$, the moment of the tetrahedral $T_{d}$ cluster with the Mn tetrahedron in the interior is equal approximately to twelve magnetic moments of the single manganese ion $\left(15.5 \mu_{\mathrm{B}}\right)$. The magnetic moment of icosahedral Al-Co clusters having the same configuration is equal to zero. The magnetic moments of the rod assembled from the icosahedral clusters with the sequence $T_{d}$ $D_{3 h}-T_{d}$ was found to be $20.5 \mu_{\mathrm{B}}$. This value permits to explain the giant magnetic moment of icosahedral and decagonal Al-Mn quasicrystals and gives the indirect evidence to the hierarchical model of the quasicrystals structure proposed by the authors recently. An arrangement of magnetic moment carriers in the interior of the aluminum shell of icosahedral clusters permits to suggest the interaction between contacting manganese ions as the main origin of the giant magnetic moment of the Al-Mn quasicrystals.
\end{abstract}

\section{Introduction}

Spin glass and giant magnetic moment are conjugated phenomena which have been observed in the alloys of noble metals with the transition 3d-metals [1-3]. The transition metal content for these effects corresponds to the region of the uniform solid solution of transition metal in the noble one with the FCC crystal structure. The indirect exchange Ruderman-Kittel-Kasuya-Yoshida (RKKY) interaction of localized magnetic moments via conducting electrons is considered as the main origin for the spin glass phenomena. In other words, random freezing of magnetic moments was explained exclusively by the features of the electron subsystem, the said features were manifested on the background of ordinary atomic FCC structure. These views are in contradiction to the observation of the spin glass behavior in the quasicrystalline Al-Mn alloys by D.P. Yang et al [4].

Quasicrystalline phases exist in the range of 17-22 at. \% $\mathrm{Mn}$ of the Al-Mn alloy system. Icosahedral quasicrystalline phase is substituted gradually by the decagonal quasicrystlline phase with increasing the manganese content thus at 22 at. \% Mn alloy consists of decagonal phase only [5]. As it was shown by D.P. Yang et al [4] both quasicrystalline phases show a spin glass behavior and values of freezing temperature and effective magnetic moment were dependent of the symmetry of the quasicrystal: respectively $3.0 \mathrm{~K}$ and $12,6 \mu_{\mathrm{B}}$ for the icosahedral phase and $7.8 \mathrm{~K}$ and $17,4 \mu_{\mathrm{B}}$ for the decagonal one. These data give evidence for the connection of the spin glass effect with the features of atomic structure but not only with the electron subsystem.

The model has been proposed recently for atomic structure of icosahedral and decagonal quasicrystalline phases in Al-Mn alloy system [6-8], the said model describes the quasicrystal structure as hierarchical joining of fragments of three-dimensional projections of the fourdimensional counterparts of icosahedron and dodecahedron, so called $\{3,3,5\}$ and $\{5,3,3\}$ polytopes. This model has no contradiction with the widely accepted description of the icosahedral quasicrystals in the framework of the mapping of the six-dimensional cubic lattice $\mathrm{D}_{6}$ onto three-dimensional Euclidian space since both four-dimensional polytopes and the six-dimensional cubic lattice are substructures of the eight-dimensional cubic diamond lattice $\mathrm{E}_{8}$. The concept of the $\mathrm{E}_{8}$ lattice (the unique maximal simple Liegh algebra) for the description of the quasicrystal structure has been proposed first by Sadoc \& Mosseri [9]. The main distinction of the model proposed in [6-8] is the use of two different clusters representing the mutual intersection of three and four icosahedra (see Figure 1) but not the single icosahedral clusters as in widely accepted models. 


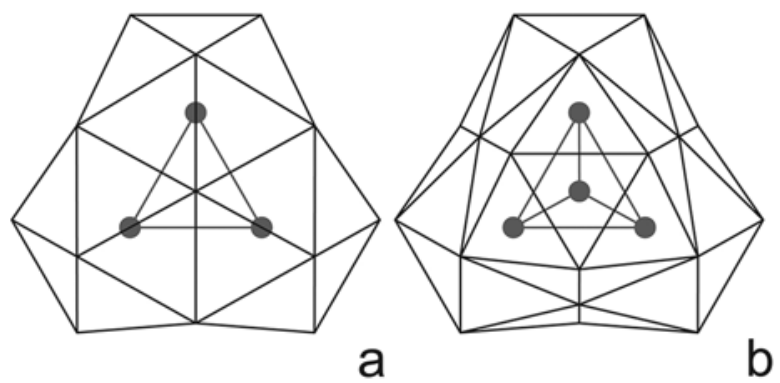

Fig. 1. Icosahedral clusters Al-Mn (Co) having $D_{3 h}\left(\right.$ a) and $T_{d \text {. }}$ (b) symmetries. Grey filled circles designate positions of $3 d-$ metals. $D_{3 h}$ clusters is a fragment of the hexagonal $\mathrm{Al}_{10} \mathrm{Mn}_{3}$ or $\mathrm{Al}_{5} \mathrm{Co}_{2}$ structure, $T_{d}$-cluster is a fragment of the cubic $\mathrm{Al}_{13} \mathrm{Cr}_{4} \mathrm{Si}_{4}\left(\mathrm{Al}_{17} \mathrm{Mn}_{4}\right)$ structure.

It must be noted three features of clusters shown in Figure 1:

1. Clusters are the fragments of some crystal structures (shown in the Figure caption), i.e. space coordinates of atoms in these clusters has been determined in experiment

2. Positions of cluster vertices are in exact coincidence with the three-dimensional projection of the fourdimensional icosahedron $(\{3,3,5\}$ polytope (see table 4 in the Coxeter monograph [10]). The trigonal cluster with the $D_{3 h}$ symmetry formed by the intersection of three icosahedra (Figure 1a) coincides with the projection started from a triangular face of the $\{3,3,5\}$ polytope, while the tetrahedral cluster (the $T_{d}$-symmetry) shown in Figure $1 \mathrm{~b}$ coincides with the projection started from a tetrahedral cell of the $\{3,3,5\}$ polytope.

3. Atoms of transition 3d-metal (TM) having magnetic moment are gathering inside the cluster thus forming a triangle or tetrahedron with a direct contact to each other. The interatomic distances for $3 \mathrm{~d}$-metals in clusters are the same as in crystal structures of the corresponding pure metal.

Joining of trigonal and tetrahedral clusters in the $T_{d^{-}}$ $D_{3 h}-T_{d}-D_{3 h}$ sequence generates a hierarchical dodecahedron (Figure 2), which is the building unit in the model for the icosahedral and decagonal quasicrystals [68].

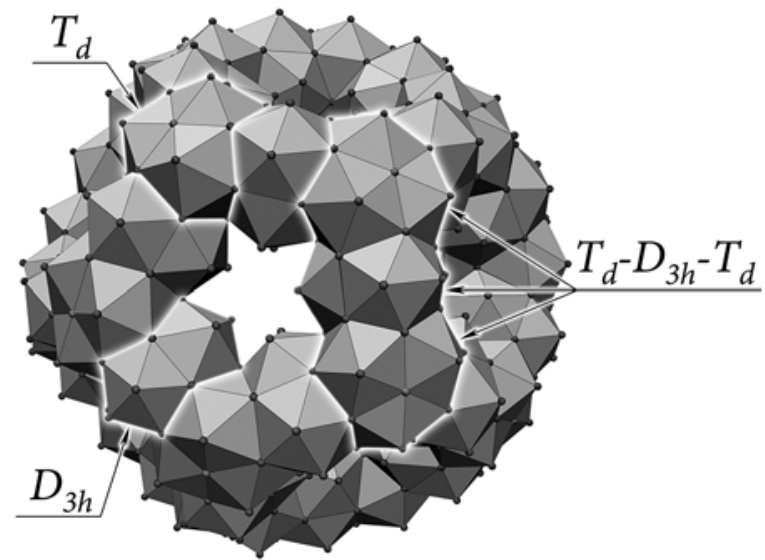

Fig. 2. An hierarchical dodecahedron assembled from rods obtained by sticking clusters in the $T_{d}-D_{3 h}-T_{d}$ sequence. This dodecahedron serves as the building unit for the quasicrystal model [6-8].
An arrangement of magnetic moment carriers in the interior of the aluminum shell of these clusters allows to suggest the interaction between contacting manganese ions as the main origin of the giant magnetic moment of the Al-Mn quasicrystals. Aiming to verify that suggestion an $a b$ initio calculation of the electron structure of the clusters in Figure 1 has been carried out in the present paper. The calculation was performed in the framework of the density functional theory (DFT).

\section{Calculation method}

For modeling the electron subsystem the DFT was selected in the present paper. DFT in the local density approximation (LDA) or in more detailed generalized gradient approximation, (GGA) allows to describe with high accuracy the equilibrium volume and stable crystal structure of pure chemical elements and their compounds. LDA utilizes the exchange-correlation energy of the uniform electron gas in each point of a system while neglecting the non-uniformity of a real charge density. In case of the non-uniform charge density the exchangecorrelation energy can deviate significantly from the uniform electron gas case. A deviation can be expressed through the gradient and space derivative of higher order of the total charge density. GGA utilizes the gradient of the charge density in order to take in account that deviation. Due to this the GGA has been used for calculation in the present paper.

One target of calculations is the total magnetic moment of all structure and of separate clusters, hence the spin polarized calculation has been utilized with the use of an $a b$ initio molecular dynamic in the framework of the electron density functional theory with the plane wave basis and PAW-potentials (projector augmentedwave potential) [11]. The VASP software code has been used for calculations [12].

The electron density was calculated for crystal structures and isolated clusters from which these structures were assembled. The shape and volume of the unit cell was fixed since the coordinates of atoms in a given structure were known with a high accuracy.

Since the VASP software code manipulates with periodic supercells only, it is not possible to prescribe in the program modeling of an isolated cluster. However, one can describe the structure in such a way when the interaction between clusters can be neglected, since interatomic forces are decreasing with interatomic distances. For this case structure can be presented as the set of separate clusters remote infinitely from each other. One can designate the unit cell with large dimensions containing a single cluster in the interior. Actually, it is impossible to designate the infinite unit cell, but the difference between the potential in a given point and the vacuum potential is sufficiently small at distances of about 1-2 nm. Bearing this consideration in mind the conditions for the computer experiment can be formulated as follows. Coordinates of atoms belonging to a cluster in the Cartesian system corresponds to atomic coordinates in the crystal unit cell while the dimensions 
of the calculation cell (domain) were set by 1-2 $\mathrm{nm}$ larger than cluster dimensions.

\section{Results and discussion}

In the framework of DFT magnetic moments were calculated for intermetallic compounds $\mathrm{Al}_{10} \mathrm{Mn}_{3}, \mathrm{Al}_{5} \mathrm{Co}_{2}$ и $\mathrm{Al}_{17} \mathrm{Mn}_{4}$ (isomorphic to $\mathrm{Al}_{13} \mathrm{Cr}_{4} \mathrm{Si}_{4}$ ). Figure 3 depicts the (0001) projections of the $\mathrm{Al}_{10} \mathrm{Mn}_{3}$ and $\mathrm{Al}_{5} \mathrm{Co}_{2}$ hexagonal structures. Both structures are formed as joining of the trigonal clusters shown in Figure 1 and having the $D_{3 h}$ symmetry. These clusters are sharing common vertices in the hexagonal plane, while along the six-fold symmetry axis [0001] clusters are joined into vertical rods by sharing common hexagonal cycles. The difference in the stoichiometry between 10:3 and 5:2 is conditional on the presence of an additional cobalt atom in the center of the trigonal prism which is filling the hollow between trigonal clusters. In order to estimate the possible influence of this additional atom having trigonal coordination calculations have been also carried out for the virtual compounds $\mathrm{Al}_{10} \mathrm{Co}_{3}$ and $\mathrm{Al}_{5} \mathrm{Mn}_{2}$.

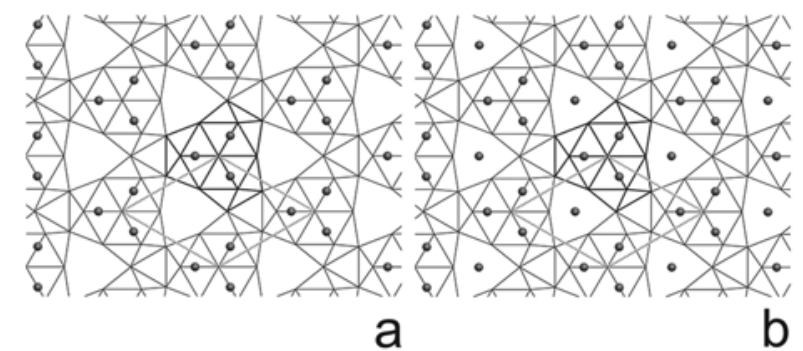

Fig. 3. View of the $\mathrm{Al}_{10} \mathrm{Mn}_{3}$ (a) and $\mathrm{Al}_{5} \mathrm{Co}_{2}$ (b) hexagonal crystal structures in the (0001) plane (unit cell boundaries are shown). $\mathrm{D}_{3 \mathrm{~h}}$ cluster is delineated by thick lines, magnetic $3 \mathrm{~d}-$ atoms designated by filled circles. Note additional 3d-atoms between $\mathrm{D}_{3 \mathrm{~h}}$ clusters (in the interior of trigonal prisms) in case of $\mathrm{Al}_{5} \mathrm{Co}_{2}$ compound.

The calculated distribution of the electron density is shown in Figures 4 and 5 for the trigonal cluster with the $D_{3 h}$ symmetry belonging to the $\mathrm{Al}_{10} \mathrm{Mn}_{3}$ intermetallic and in Figure 6 for the tetrahedral $T_{d}$ cluster belonging to the $\mathrm{Al}_{17} \mathrm{Mn}_{4}$ intermetallics. The results of magnetic moment calculation are given in the Table 1.

The data given in the Table 1 show that the magnetic moment of the manganese triangle corresponds to three moments of a single manganese ion.

The total moment both of the compound and isolated cluster is equal to zero for case of cobalt as the transition metal with the same cluster configuration. The calculation for the virtual $\mathrm{Al}_{10} \mathrm{Co}_{3}$ compound shows that an elimination of the "extra" cobalt atom out from the trigonal prism keeps the zero magnetic moment unchanged while the insertion of manganese atom into the trigonal prism (a virtual compound $\mathrm{Al}_{5} \mathrm{Mn}_{2}$ ) results in the significant increase of the magnetic moment. The zero magnetic moment in cobalt compounds points to significant differences between cobalt and manganese interactions with aluminum in these compounds.

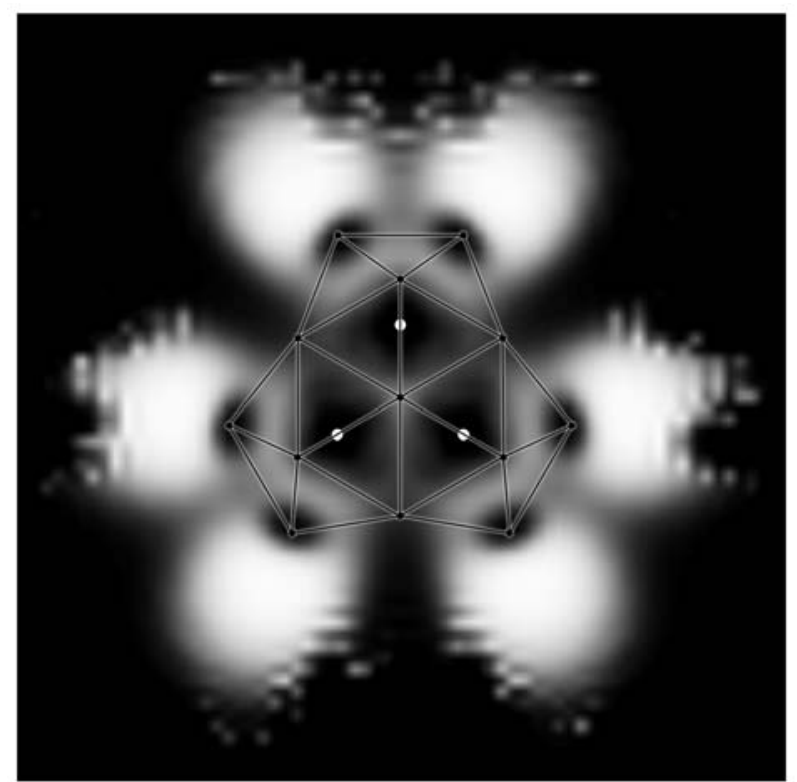

Fig. 4. Equatorial section of the electron density distribution calculated for the $D_{3 h}$ cluster of the $\mathrm{Al}_{10} \mathrm{Mn}_{3}$ compound in the plane of Mn triangle (an equatorial section). White color is the maximal density, black background is zero level.

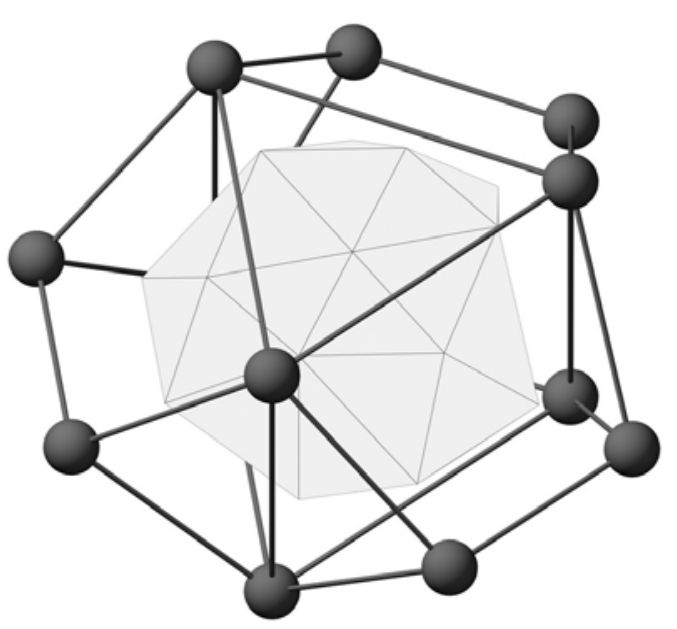

Fig. 5. The distribution of the electron density maxima (filled spheres) around a trigonal $D_{3 h}$ cluster of the $\mathrm{Al}_{10} \mathrm{Mn}_{3}$ compound. Three-dimensional picture has been reconstructed from different sections of the calculated electron density. 


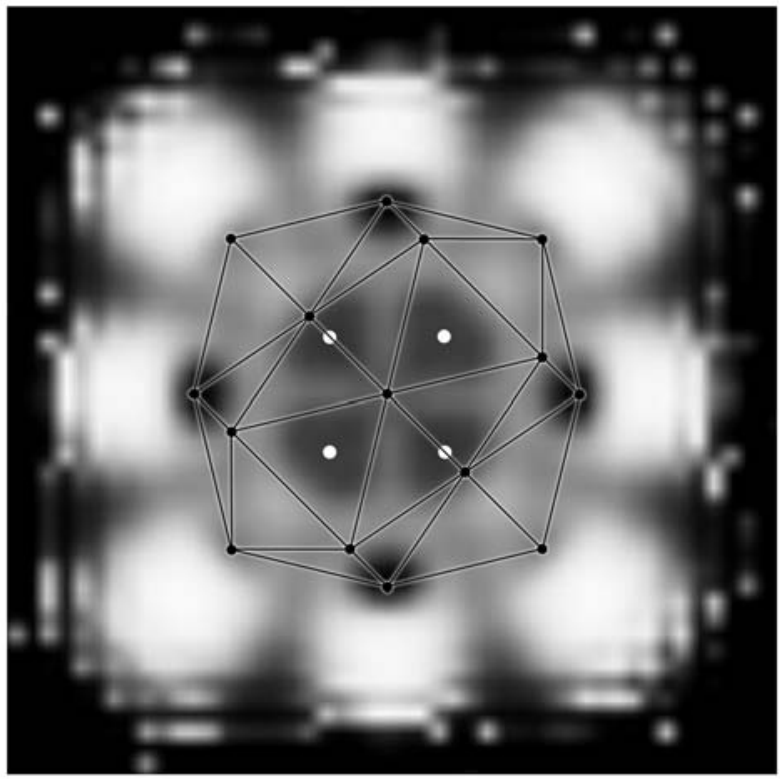

Fig. 6. Equatorial section of the electron density distribution calculated for the $T_{d}$ cluster of the $\mathrm{Al}_{17} \mathrm{Mn}_{4}$ compound. White color is the maximal density, black background is a zero level. Magnetic atoms (forming a tetrahedron) designated by white filled circles.

In case of the tetrahedral cluster of magnetic atoms the total magnetic moment corresponds to twelve magnetic moments of a single manganese ion. As a crude approximation one can say that the total moment of the tetrahedral cluster corresponds to the moment of four triangular faces of a tetrahedron (since the total moment of the manganese triangle was found to be equal approximately to three moments of the single manganese ion).

The discovered increasing of the magnetic moment in both trigonal and tetrahedral aluminum-manganese clusters allows verifying two hypotheses:

1) The giant magnetic moment observed in Al-Mn quasicrystal is caused by the presence of the magnetic atom clusters in the quasicrystal structure;

2) The atomic structure of icosahedral and decagonal quasicrystals does indeed assembled from clusters shown in Figures 1 and 2.

Both hypotheses states above are interdependent: if the second is true, so the first is supported.
For checking of both hypotheses one must calculate the magnetic moment for the hierarchical dodecahedron shown in Figure 2. This hierarchical dodecahedron contains 790 atoms so the computer calculation of its total magnetic moment is not possible since it requires too much memory resources. Due to this reason the magnetic moment calculations have been carried out only for fragments of the dodecahedral cluster, those fragments are joining of clusters with $D_{3 h}$ and $T_{d}$ symmetries.

The hierarchical rod (an edge of the dodecahedron) is delineated in Figure 2. This rod was generated by sticking the icosahedral clusters in the sequence of $T_{d}-D_{3 h}-T_{d}$. The calculation of magnetic moment for this sequence results in the value of 20.5 Bohr magnetons. As can be seen the calculated moments of the tetrahedral cluster (Table 1) and a rod formed by two tetrahedral cluster separated by the trigonal cluster are in good agreement with the giant magnetic moments observed in [4] for the Al-Mn quasicrystals.

The hierarchical dodecahedron shown in Figure 2 contains $20 \times 4+30 \times 3=170$ manganese atoms. According to estimates by [4] the observed giant magnetic moments of Al-Mn quasicrystalline phases correspond to the clusters containing 116 (the icosahedral phase) and 126 (the decagonal phase) magnetic moments. In other words, one can consider the results of our calculation as an evidence for both stated hypotheses.

The enhanced magnetic moments of the tetrahedral configuration formed by atoms of transition 3d-metals which were observed in the present paper can be probably the foundation for some well-known experimental facts: 1) the ferromagnetic quasicrystals have been observed in the Al-Mn-Si [13] and Mn-Al-Ge$\mathrm{B}$ [14] systems, in which there are a high probability for the presence of clusters having $T_{d}$ and $D_{3 h}$ symmetries; 2) the sharp maxima of the diamagnetic susceptibility and Hall constant in the $\mathrm{Cu}-\mathrm{Zn}$ alloy system coincides exactly to the $\gamma$-brass composition $\mathrm{Cu}_{5} \mathrm{Zn}_{8}$ having the crystal structure composed from the $T_{d}$-clusters shown in Figure 1 (so called $\gamma$-brass clusters) [15]. It is possible that the tetrahedral coordination of the magnetic moment carriers and their icosahedral environment favour to the emergence of the giant magnetic moment.

Table 1. The results of magnetic moment calculation.

\begin{tabular}{|c|c|c|c|c|}
\hline Compound & $\begin{array}{c}\text { Al-TM cluster } \\
\text { symmetry }\end{array}$ & $\begin{array}{c}\text { TM cluster } \\
\text { configuration }\end{array}$ & $\begin{array}{c}\text { Magnetic moment of } \\
\text { the compound, } \boldsymbol{\mu}_{\mathbf{B}}\end{array}$ & $\begin{array}{c}\text { Magnetic moment of } \\
\text { isolated cluster Al- } \\
\mathbf{T M}, \boldsymbol{\mu}_{\mathbf{B}}\end{array}$ \\
\hline $\mathrm{Al}_{10} \mathrm{Mn}_{3}$ & $D_{3 h}$ & triangle & 4.6 & 4.4 \\
\hline $\mathrm{Al}_{10} \mathrm{Co}_{3}$ & $D_{3 h}$ & triangle & 0 & 0 \\
\hline $\mathrm{Al}_{5} \mathrm{Co}_{2}$ & $D_{3 h}$ & triangle & 12.6 & 12.6 \\
\hline $\mathrm{Al}_{5} \mathrm{Mn}_{2}$ & $D_{3 h}$ & triangle & - & 15.5 \\
\hline $\mathrm{Al}_{17} \mathrm{Mn}_{4}$ & $T_{d}$ & tetrahedron & \\
\hline
\end{tabular}




\section{Conclusions}

$A b$ initio calculations of magnetic moments for icosahedral clusters contained in crystal structures $\mathrm{Al}_{10} \mathrm{Mn}_{3}, \quad \mathrm{Al}_{5} \mathrm{Co}_{2}, \quad \mathrm{Al}_{17} \mathrm{Mn}$ performed in the DFT framework show increasing of magnetic moment: the AlMn cluster having the trigonal $D_{3 h}$ symmetry shows the moment of three magnetic moments of a single manganese ion $\left(4.4 \mu_{\mathrm{B}}\right)$, the moment of the tetrahedral $T_{d}$ cluster is equal approximately to twelve magnetic moments of the single manganese ion $\left(15.5 \mu_{\mathrm{B}}\right)$. The observed values of magnetic moment correspond respectively to the triangular and tetrahedral configurations of manganese clusters with direct contacts Mn-Mn.

The magnetic moment of icosahedral Al-Co clusters having the same configuration is equal to zero.

The magnetic moments of the rod assembled from the icosahedral clusters with the sequence $T_{d}-D_{3 h}-T_{d}$ was found to be $20.5 \mu_{\mathrm{B}}$. This value allows explaining the giant magnetic moment of icosahedral and decagonal AlMn quasicrystals and gives the indirect evidence to the hierarchical model of the quasicrystals structure put forward by authors before.

\section{Acknowledgments}

This work was fulfilled with the financial support from the RFBR of Russian Academy of Science, grants 08-0201177 and 10-02-00602, computer calculations has been fulfilled with the supercomputer MVS6K provided by Joint SuperComputer Center (JSCC), Moscow, Russia.

\section{References}

1. Mydosh I.A. Spin Glasses, an experimental introduction. London: Taylor and Francis, 1993.

2. Binder K., Yong A.P. Spin glasses: Experimental facts, theoretical concepts, and open questions//Rev. Mod. Phys. 1986. V.58. \#4. P.801-976.

3. Fisher K.H., Hertz J.A. Spin Glasses. England: Cambridge University, Cambridge, 1991. 418 p.

4. Yang D.P., Hines W.A., Clark W.G., Machado F.L.A., Azevedo L.A., Giessen B.C., Quan M.X. Magnetization study of the I-A180Mn20 and T$\mathrm{Al}_{78} \mathrm{Mn}_{22}$ quasicrystalline phases//Journal of Magnetism and Magnetic Materials. 1992. V.109. P.1-6.

5. Bendersky L. Quasicrystal with One-Dimensional Translational Symmetry and a Tenfold Rotation Axis//Phys. Rev. Lett. 1985. V.55. P.1461-1463.

6. Kraposhin V.S. Assembly of an Icosahedral Quasicrystal from Hierarchic Atomic Clusters//Crystallographic reports. 1996. V.41. P.371-380.

7. Kraposhin V.S. Assembly of an Icosahedral Quasicrystal from Hierarchic Atomic Clusters:
Decagonal Symmetry//Crystallographic reports. 1999. V.44. P.927-937.

8. Kraposhin V.S., Talis A.L., Lam Ha Thanh, Dubois J.-M. Model for the transformation of an icosahedral phase into a B2 crystalline phase //J. Phys.: Condens. Matter. 2008. V.20. P.235215 (8pp).

9. Sadoc J.-F., Mosseri R. The E8 lattice and quasicrystals//J. Non-Cryst.Solids 1993. V.153/154. P.247-252.

10. Coxeter H. S .M. 1983 Regular Polytopes (New York: Dover).

11. P.E. Blochl Phys. Rev. B 50, 17953 (1994).

12. G. Kresse and J. Furthmuller, Phys. Rev. B 54, 11169 (1996)

13. Dunlap R.A., McHenry M.E., Srinivas V., Bahadur D., O'Handley R.C. Ferromagnetism in icosahedral Al-Mn-Si alloys//Phys. Rev. B. 1989. V. 39. P. 48084811.

14. Yokoyama Y., Inoue A. Ferromagnetic Mn-Based Decagonal Alloys at Room Temperature//Japan. J. Appl. Phys. 1996. V. 35. P. 3533-3534.

15. Jones H. The Theory of Alloys in the $\gamma$-Phase//Proc. Roy. Soc. London. 1934. V. A144. P.225-234. 